\title{
Real-Time Microgravimetric Quantification of Cryptosporidium parvum in the Presence of Potential Interferents
}

\author{
Revised and resubmitted to: \\ Water Research \\ March 12, 2009 \\ CHARLES POITRAS, JULIEN FATISSON, and NATHALIE TUFENKJI* \\ Department of Chemical Engineering, McGill University, Montreal, Quebec H3A \\ 2B2, Canada
}

* Corresponding Author. Phone: (514) 398-2999; Fax: (514) 398-6678; E-mail: nathalie.tufenkji@mcgill.ca 


\begin{abstract}
The quartz crystal microbalance with dissipation monitoring (QCM-D) is used to develop a biosensor for detection of viable Cryptosporidium parvum (C. parvum) in water matrices of varying complexity. In a clean environment, a good log-log linear response is obtained for detection of $C$. parvum in aqueous suspensions with oocyst concentrations from $3 \times 10^{5}$ to $1 \times 10^{7}$ oocysts/mL. C. parvum detection is slightly affected by the presence of dissolved organic acids, likely due to steric stabilization and/or masking of the antibodies/antigens by adsorbed molecules. Colloidal contaminants generally have a greater influence as biosensor interferents, whereby the presence of model latex microspheres, E. faecalis, or E. coli, led to decreases in biosensor response of up to $64 \%, 40 \%$, and $20 \%$, respectively.
\end{abstract}

Keywords: QCM-D, C. parvum, interference, dissipation slope, biosensor 


\section{Introduction}

Cryptosporidium parvum (C. parvum) has emerged as one of the most important microbial pathogens for drinking water safety and is associated with a high risk of waterborne illness (Rose, 1997). The protozoan parasite C. parvum is commonly transmitted via the faeces of infected mammalian hosts in the form of an oocyst which is highly resistant to environmental stresses (Smith, 1992). Ingestion of a small number of viable oocysts (as little as 10) can lead to cryptosporidiosis, a diarrheal disease that is potentially lethal for immunosuppressed individuals (Casemore et al., 1997, Rose, 1997). Several outbreaks of cryptosporidiosis related to drinking water contamination have been reported in Europe and North America over the past 20 years (Lisle and Rose, 1995, Smith and Perdek, 2004). The public health risk posed by C. parvum underscores the need to develop rapid and reliable sensors for detection of the parasite in potable water supplies.

Various approaches have been proposed for detection of $C$. parvum in water samples, including conventional microscopy, immunological assays, flow cytometry, and nucleic-acid based methods (Jex et al., 2008). These methods require varying extents of sample preparation, concentration, and/or labeling, and exhibit different levels of detection. For example, the commonly used US EPA method 1623 requires filtration, immunomagnetic separation of the oocysts, and an immunofluorescence assay with confirmation via differential interference contrast microscopy (Jex et al., 2008). Recently, a piezoelectric-excited millimeter-sized cantilever (PEMC) sensor has successfully been used to detect C. parvum oocysts at concentrations as low as 100 oocysts/mL in less than 1 min (Campbell and Mutharasan, 2008). The quartz crystal microbalance (QCM) functions in a manner analogous to PEMC biosensors 
but has not previously been considered for detection of C. parvum. Hence, it is of interest to evaluate the potential use of QCM in direct, real-time detection of C. parvum.

A major challenge associated with detection of target microorganisms is interference by dissolved substances or particulates present in the water samples. Organic matter, microbial exudates, and colloidal particles (including a broad range of microorganisms) are commonly found in untreated surface waters and groundwater supplies and may interfere with biosensor performance. However, few studies of biosensor development consider the potential influence of dissolved and/or colloidal contaminants on pathogen detection in complex matrices (Campbell et al., 2007, Edgar et al., 2006, Kang et al., 2008, Subramanian et al., 2006, Waswa et al., 2006). Some researchers have evaluated biosensor interference by non-target organisms (Lee et al., 2005, Su and Li, 2005, Su and Li, 2004, Vaughan et al., 2001), however, the potential influence of non-biological colloids using model particles has not been examined in previous studies of biosensor development. Another important difficulty in evaluating the occurrence of waterborne pathogens such as $C$. parvum is the relatively low environmental concentrations in contaminated water supplies ( 0.1-10 oocysts/L) (Mons et al., 2009).

The purpose of this study is to examine the potential interference of a biosensor based on a quartz crystal microbalance with dissipation monitoring (QCM-D) by natural organic matter (NOM) and model colloids (including bacteria) that may be found in natural waters. Antibodies immobilized onto a gold coated QCM-D crystal were used for rapid detection of viable $C$. parvum oocysts over a range of concentrations. Experiments conducted in the presence of NOM demonstrate some interference, whereas colloidal contaminants generally yield larger decreases in the biosensor response. 


\section{Materials and Methods}

2.1. Reagents. Mouse monoclonal immunoglobulin $\mathrm{M}$ (IgM), specific to C. parvum, was obtained from Waterborne Inc., New Orleans, LA. Bovine serum albumin (BSA) and phosphate buffer saline (PBS) tablets were purchased from Sigma-Aldrich. Suwannee river humic acid (SRHA) and Suwannee river fulvic acid (SRFA) standards (International Humic Substances Society, St. Paul, MN) were dissolved in PBS. Surfactant-free polystyrene latex microspheres (1.5 $\mu \mathrm{m}$ diameter) with sulfate functional groups (Interfacial Dynamics Microspheres and Nanospheres, Eugene, OR) were suspended in PBS at a concentration of $5 \times 10^{6}$ particles $/ \mathrm{mL}$. Denatured alcohol (Fisher Scientific, Canada), Hellmanex (Hellma, Thornhill, ON), hydrogen peroxide (Fisher), and ammonia (LabChem Inc., ON) were used to clean the crystal surfaces after each experiment. Ultrapure deionized (DI) water was used to prepare all PBS solutions (Biolab).

2.2. Preparation of Oocysts and Bacteria. Viable oocysts were obtained from the Sterling Parasitology Laboratory (SPL) at the University of Arizona. The oocysts were shed from a calf infected with the Iowa isolate from Dr. Harley Moon (National Animal Disease Center, Ames, Iowa). Oocysts were purified (at SPL) using discontinuous sucrose and cesium chloride centrifugation gradients and stored (in the dark at $\left.4^{\circ} \mathrm{C}\right)$ in an antibiotic solution $(0.01 \%$ Tween $20,100 \mathrm{U}$ of penicillin, and $100 \mu \mathrm{g}$ of gentamicin per $\mathrm{mL}$ ). Before conducting experiments, oocysts were pelleted by centrifugation (9,300g for $2 \mathrm{~min}$, IEC MicroCL 17R, Thermo) and the supernatant was replaced with $1 \mathrm{~mL}$ PBS. This step was repeated twice to remove any trace of the antibiotic solution. Purified oocysts were diluted to the desired concentration in PBS (pH 
7.4) prior to the QCM-D experiments. The oocyst concentration was determined using a cell counting chamber (Hawksley Medical and Lab Equipment, Lancing, UK).

Escherichia coli (E. coli) O157:H7 (ATCC 700927) and Enterococcus faecalis (E. faecalis) (ATCC 29212) were used as representative interfering pathogenic organisms. E. coli O157:H7 is a Gram-negative bacterium while E. faecalis is a Gram-positive organism. Pure cultures were maintained at $-80^{\circ} \mathrm{C}$ in Luria-Burtani (LB) Lennox broth (Fisher Scientific, Canada) (20 g/L) containing 15\% glycerol. Pure cultures were then streaked onto LB agar followed by $24 \mathrm{~h}$ incubation at $37^{\circ} \mathrm{C}$. For interference experiments using bacteria, colonies from a fresh plate were used to inoculate $150 \mathrm{~mL}$ of $\mathrm{LB}$ broth (in a $500 \mathrm{~mL}$ baffled flask). Liquid cultures were incubated at $37^{\circ} \mathrm{C}$ for $18 \mathrm{~h}$ at $200 \mathrm{rpm}$. The bacterial suspension was harvested by centrifugation (Sorvall RC6, Thermo Scientific, Waltham, MA) at 5860g in a SS34 rotor (Kendro, Thermo Scientific, Waltham, MA) at $4^{\circ} \mathrm{C}$. The bacterial pellet was re-suspended in PBS at $\mathrm{pH}$ 7.4. The centrifugation and re-suspension steps were repeated one additional time to remove any traces of growth media. The resulting bacterial cell concentration (total cells) was determined using the same cell counting chamber (Hawksley Medical and Lab Equipment).

For interference experiments, the following suspensions were prepared: (i) mixture of $C$. parvum $\left(5 \times 10^{6}\right.$ oocysts $\left./ \mathrm{mL}\right)$ and E. coli $\left(5 \times 10^{6}\right.$ cells $\left./ \mathrm{mL}\right)$; (ii) mixture of $C$. parvum $\left(5 \times 10^{6}\right.$ oocysts $/ \mathrm{mL})$ and E. faecalis $\left(5 \times 10^{6}\right.$ cells $\left./ \mathrm{mL}\right)$; (iii) mixture of $C$. parvum $\left(5 \times 10^{6}\right.$ oocysts $\left./ \mathrm{mL}\right)$ and latex microspheres $\left(5 \times 10^{6}\right.$ particles $\left./ \mathrm{mL}\right)$; (iv) C. parvum $\left(5 \times 10^{6}\right.$ oocysts $\left./ \mathrm{mL}\right)$ in PBS supplemented with $1 \mathrm{mg} / \mathrm{L}$ SRHA or SRFA.

2.3. Detection of Cryptosporidium using $Q C M-D$. A quartz crystal microbalance with dissipation monitoring (QCM-D) was used as a platform to monitor binding of $C$. parvum and/or 
the interfering agents to an antibody covered gold coated crystal surface. QCM-D differs from traditional QCM in that it gives insight into the viscoelastic properties of the adsorbed mass. The fundamental concept of the QCM-D technology is that a "soft" mass adhered to the crystal surface will not fully couple to the crystal's oscillation and thus will dampen it (Hook, 1997). The energy dissipation factor $D$ represents the sum of all processes that induce energy losses in the oscillating system (Hook, 1997). The E4 QCM-D unit (Q-Sense AB, Västra Frölunda, Sweden) consists of a measurement platform that can hold 4 sensor flow chambers with a volume of $40 \mu \mathrm{L}$ each. The flow chambers were maintained at $20^{\circ} \mathrm{C}$ during experiments. Clean gold-coated QCM-D crystals (QSX 301, $5 \mathrm{MHz}$, AT-cut, Q-Sense AB) were mounted inside the flow modules. PBS ( $\mathrm{pH} 7.4)$ was injected for $10 \mathrm{~min}(50 \mu \mathrm{L} / \mathrm{min})$ using a peristaltic pump (Reglo-Digital IPC-N4, Ismatec SA, Glattbrugg, Switzerland) to ensure that stable baselines in frequency $(f)$ and energy dissipation $(D)$ shifts were achieved. A suspension of anti-C. parvum antibodies $(10 \mu \mathrm{g} / \mathrm{mL})$ in PBS was injected into each flow chamber for 20 min at a flow rate of $50 \mu \mathrm{L} / \mathrm{min}$. The pump was then stopped for a duration of $60 \mathrm{~min}$ to allow time for the antibody to physically adsorb to the gold surface. This step was followed by a 20 min PBS rinse under flow to remove any unbound antibody. Next, a BSA solution in PBS $(10 \mathrm{mg} / \mathrm{mL})$ was injected for $20 \mathrm{~min}(50 \mu \mathrm{L} / \mathrm{min})$ to block non-specific sites, and the pump was stopped for $60 \mathrm{~min}$ before a 20 min PBS rinse to remove any unbound BSA. The prepared sensors were then ready for detection of the target organism. A suspension of $C$. parvum or mixture of $C$. parvum and bacteria/microspheres was then injected for a period of $10 \min (50 \mu \mathrm{L} / \mathrm{min})$ after which the pump was stopped for $60 \mathrm{~min}$ to allow sufficient time for oocyst attachment to the antibody-coated surface. A final 20 min PBS rinse was then performed. Shifts in the crystal frequency and energy dissipation for the first overtone were continuously monitored at each experimental step 
using the Q-Soft software (Q-Sense AB). Following each experiment, the QCM-D crystals were imaged (see section 2.4) then cleaned using the following procedure: the crystals were (i) sonicated for 5 minutes in denatured alcohol (ii), sonicated for $5 \mathrm{~min}$ in the presence of $2 \%$ Hellmanex, (iii) rinsed thoroughly with DI water, (iv) soaked in a hot mixture $\left(85^{\circ} \mathrm{C}\right)$ of $30 \%$ $\mathrm{H}_{2} \mathrm{O}_{2}$, ammonia, and DI water, at a 1:1:5 ratio, (v) rinsed with DI water, and (vi) dried with ultrahigh purity (UHP) $\mathrm{N}_{2}$. Each experiment was conducted with a clean gold-coated crystal and required the antibody adsorption step prior to the detection step.

2.4. Optical and Electron Microscopy. Following each QCM-D experiment, the gold-coated crystals were gently rinsed with DI water, and dried under a stream of UHP $\mathrm{N}_{2}$. The crystals were then imaged using optical microscopy (IX-71, Olympus) and images analyzed using ImageJ software.

High resolution images of $C$. parvum oocysts were obtained using a Hitachi S-4700 Field Emission Gun Scanning Electron Microscope (FEG-SEM). A suspension of oocysts (10 $\mu \mathrm{L})$ was pipetted onto a gold coated glass slide and left to sit for $1 \mathrm{~h}$ to allow oocyst binding. The slides were then rinsed with PBS, and the oocysts were fixed twice overnight with $2.5 \%$ glutaraldehyde in PBS ( $\mathrm{pH}$ 7.4). The samples were then dehydrated using ethanol/water solutions of increasing ethanol content from $30 \%$ to $100 \%$ and amyl acetate/ethanol solutions, with increasing amyl acetate content from $25 \%$ to $100 \%$. Dehydration was followed by a critical point drying step (LADD Research Industries) and the slides were finally coated with Au/Pd prior to imaging. 


\section{Results and Discussion}

3.1. Cryptosporidium Detection in a Clean Water Matrix. Preliminary experiments were carried out to evaluate two approaches for immobilization of anti-C. parvum antibodies to the gold coated quartz crystal surface. Direct physisorption of the pentamer-shaped antibodies was conducted as described in section 2.3. Immobilization of antibodies via a cysteamine selfassembled monolayer (as described in (Poitras and Tufenkji, 2009)) was also considered. The $f$ and $D$ shifts measured after binding of $C$. parvum oocysts to the physisorbed or chemically immobilized antibodies were nearly identical. Hence, for simplicity, physisorption was selected as the method for antibody immobilization on the gold coated crystal surface.

Shifts in the resonance frequency $(f)$ and dissipation factor $(D)$ were monitored during injection of the antibody solution (10 $\mu \mathrm{g} / \mathrm{mL})$ into the QCM-D flow chambers (Figure 1). At the end of phase I, the resonance frequency $(f)$ had reached a stable value of $-45.81 \pm 0.05 \mathrm{~Hz}$, indicating an increase in adhered mass at the crystal surface. Correspondingly, at the end of phase I, the energy dissipation factor $(D)$ had reached a plateau at $2.42 \pm 0.01 \mathrm{DU}$. 
Figure 1. QCM-D measurements of the $1^{\text {st }}$ overtone (a) frequency and (b) dissipation shifts during physisorption of anti-C.parvum antibodies onto gold coated quartz crystals (phase I) followed by BSA adsorption (phase II).
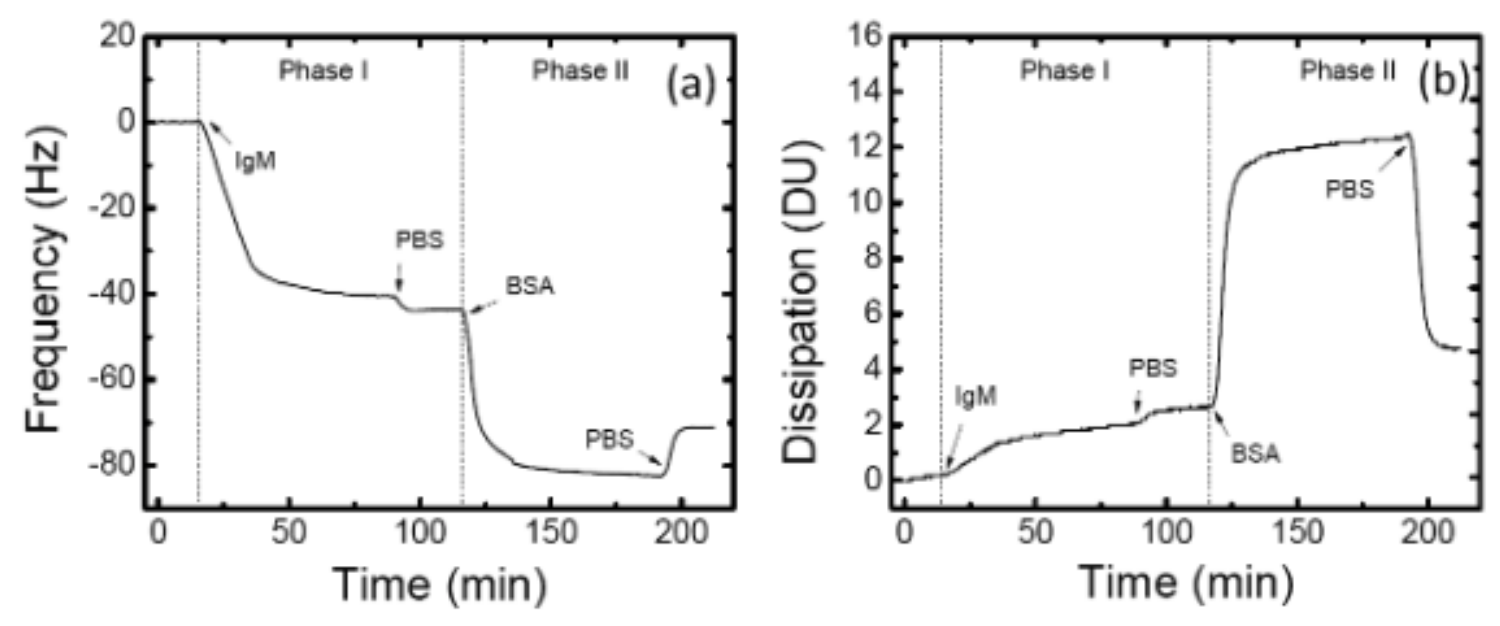
To reduce non-specific binding of organisms to the antibody layer, BSA was used as a blocking agent. During injection of BSA into the flow modules (Phase II in Figure 1), a negative shift in $f$ and a positive shift in $D$ were observed, as previously shown for protein adsorption (Dolatshahi-Pirouz et al., 2008, Hook et al., 2002, Nimeri et al., 1998). During injection of BSA, the measured shift in the resonance frequency $(\triangle f)$ was $-20.51 \pm 0.07 \mathrm{~Hz}$, corresponding to an increase in adhered mass onto the crystal. During phase II, the total shift in the energy dissipation factor $(\triangle D)$ was $1.46 \pm 0.01$ DU. Model calculations of the QCM-D data using the QTools software (Q-Sense AB) predict a thickness of $3.40 \pm 0.01 \mathrm{~nm}$ for the BSA layer, which is slightly greater than the shorter ellipsoidal axis of an albumin molecule $(3 \mathrm{~nm} \times 8.2 \mathrm{~nm} \times 8.5$ nm) (Hook et al., 1998). In these calculations, the density of the antibody layer was estimated as $1.35 \mathrm{~g} / \mathrm{cm}^{3}$ (Hook et al., 2001, Hook et al., 2002). The model calculations should be considered with caution however since the adsorbed layer can also be composed of rigidly associated water. Moreover, BSA likely does not form a uniform monolayer above the layer of antibodies as assumed in the model calculations (Tencer et al., 2007). Nevertheless, this result suggests that BSA conformation upon adsorption onto the crystal is more likely to be horizontal than vertical as has been previously reported for deposition onto polystyrene and silica surfaces (Green et al., 1997, Kim and Somorjai, 2003). The calculated mass of BSA on the crystal surface (459 $\mathrm{ng} / \mathrm{cm}^{2}$ ) is in accordance with previous studies (Hook et al., 2002, Lubarsky et al., 2007). (Hook et al., 2002) obtained a value of $333 \mathrm{ng} / \mathrm{cm}^{2}$ albumin coverage from a much more dilute solution (80 $\mu \mathrm{g} / \mathrm{mL})$. Our result is also comparable to that obtained by (Lubarsky et al., 2007) who reported a BSA thickness of nearly $3 \mathrm{~nm}$, corresponding to a mass of $405 \mathrm{ng} / \mathrm{cm}^{2}$.

Figure 2 shows an SEM image of an intact $C$. parvum oocyst on the surface of a gold coated slide. The oocyst is nearly $3 \mu \mathrm{m}$ in diameter and is spherical in shape. Representative $f$ 
and $D$ shifts measured during injection of a suspension of $C$. parvum oocysts are presented in Figure 3. When oocysts are injected over the antibody covered gold coated crystal, both the resonance frequency $(f)$ and energy dissipation factor $(D)$ increase with time. According to Sauerbrey (Sauerbrey, 1959), an increase in attached mass on the crystal surface is reflected by a decrease in the resonance frequency of the crystal. However, the data in Figure 3 show an opposite behavior whereby a positive shift in $f$ is detected during C. parvum injection. Previous QCM studies using Salmonella, E. coli, Listeria, and metal oxide nanoparticles (Fatisson et al., 2009, Poitras and Tufenkji, 2009, Su and Li, 2005, Vaughan et al., 2001) have suggested that the observed positive frequency shifts may be attributed to the accumulation of weakly attached particles at the liquid-crystal interface or the formation of thick viscoelastic films on the crystal surface, while negative frequency shifts are normally expected when stronger interactions are involved. Both the measured $f$ and $D$ shifts increase with increasing oocyst concentration while the control experiment (PBS with no oocysts) exhibits no change in $f$ or $D$. Hence, the raw data presented in Figure 3 suggests that the QCM-D measurements provide real-time sensing of $C$. parvum oocysts in suspension. 
Figure 2. FEG-SEM image of a C. parvum oocyst $(20,000 \times$ magnification) adhered onto a gold coated glass slide.

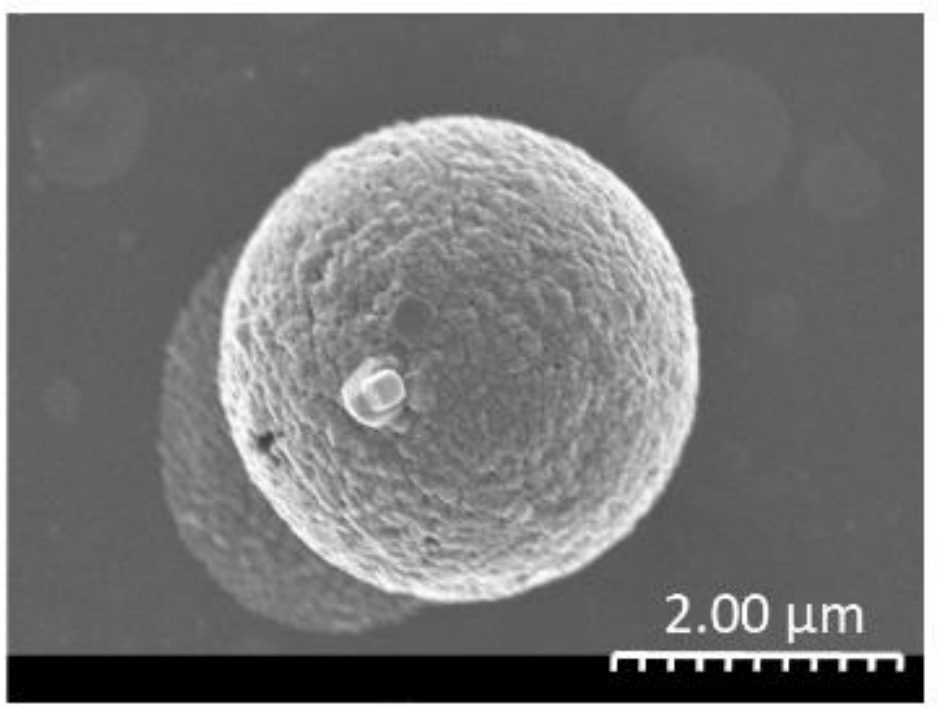

Figure 3. Representative QCM-D measurements of the $1^{\text {st }}$ overtone (a) frequency and (b) dissipation shifts for binding of $C$. parvum to antibody modified gold coated quartz crystal. Phase I: baseline in PBS; Phase II: C. parvum oocysts suspended in PBS (pH 7.4) are injected at different concentrations: $10^{7}$ oocysts $/ \mathrm{mL}$ (solid lines), $5 \times 10^{6}$ oocysts $/ \mathrm{mL}$ (dashed lines), $10^{6}$ oocysts/mL (dotted lines) and no oocyst (dashed dotted lines).
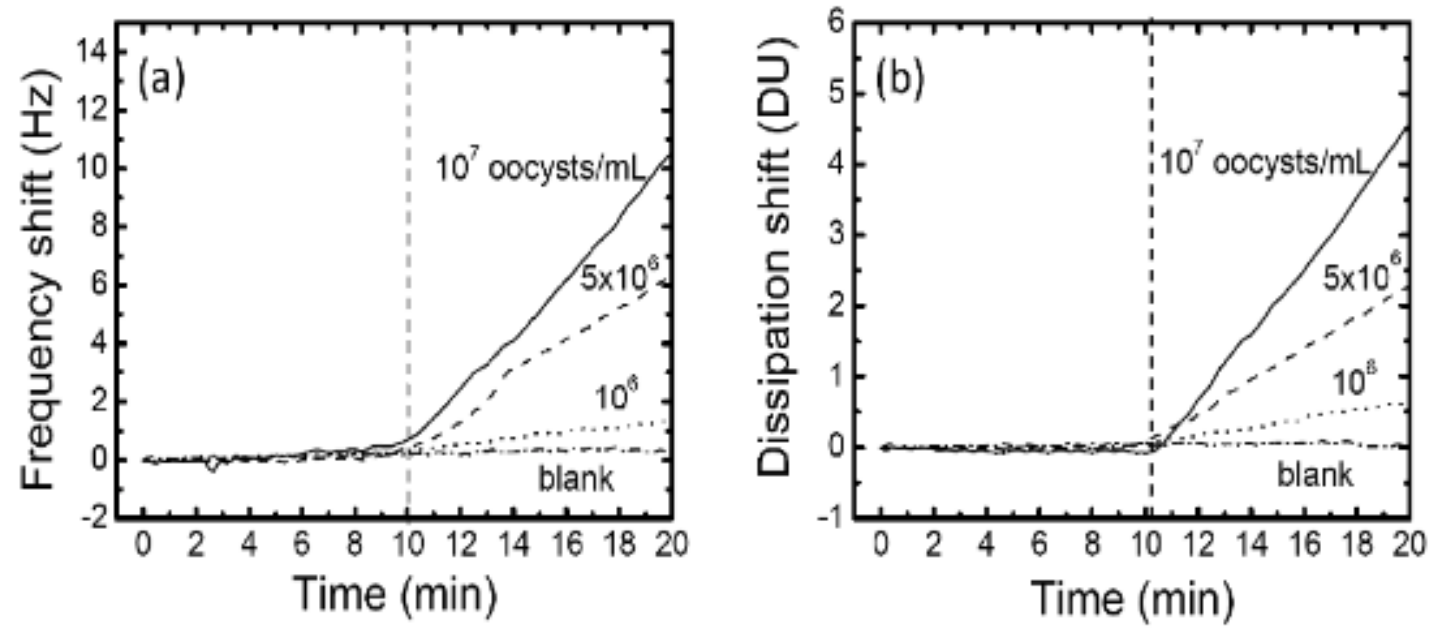
Other studies of QCM based biosensors (Mao et al., 2006, Shen et al., 2007, Su and Li, 2005, Su and Li, 2004, Vaughan et al., 2001, Wong et al., 2002) have reported dose-response relationships based on the measured $f$ shift after stabilization of the QCM signal. In our earlier work, (Poitras and Tufenkji, 2009) we demonstrated the value of calculating the initial slopes in the measured $f$ and $D$ shifts as a function of time as important biosensor signals. The values of $f_{\text {slope }}$ and $D_{\text {slope }}$ can represent the rate of change of mass or energy loss at the crystal surface which are related to the rate of particle/microbe attachment. One advantage of using these parameters as biosensor responses is that one does not need to wait for the signal to stabilize (i.e., reach a plateau). Hence, the measurement of the initial $f_{\text {slope }}$ and $D_{\text {slope }}$ can provide a rapid means to evaluate the biosensor response (Poitras and Tufenkji, 2009) as only the first few minutes of detection are needed.

Figure 4 presents the calculated values of the initial $f_{\text {slope }}$ and $D_{\text {slope }}$ when suspensions of C. parvum at different oocyst concentrations were injected into the QCM-D flow chambers. The open symbols represent measurements obtained using suspensions of C. parvum in PBS alone. In the absence of potential interfering agents, the calculated values of $f_{\text {slope }}$ exhibit a log-log linear increase $\left(R^{2}=0.99\right)$ as a function of oocyst concentration, from a concentration of $3 \times 10^{5}$ oocysts $/ \mathrm{mL}$ to a maximum tested limit of $10^{7}$ oocysts/mL (Fig. 4a). Likewise, the data show a $\log -\log$ linear increase in $D_{\text {slope }}\left(R^{2}=0.99\right)$ over the same range of injected oocyst concentrations (Fig. 4b). Below a concentration of $3 \times 10^{5}$ oocysts $/ \mathrm{mL}$, the $f$ and $D$ shifts could not be distinguished from the baseline signals and the measured drift of the instrument; hence, these data can not be included in Fig. 4 which is in a log-log scale. The maximum oocyst concentration evaluated was $10^{7}$ oocysts $/ \mathrm{mL}$. In our earlier work, we found that the $D_{\text {slope }}$ was a better choice of biosensor response signal than the $f_{\text {slope }}$ (Poitras and Tufenkji, 2009) for detection 
of E. coli $\mathrm{O} 157: \mathrm{H7}$. In the present study with $C$. parvum oocysts, it can be noted that the response behavior of the biosensor is similar for both the $f_{\text {slope }}$ and the $D_{\text {slope }}$.

Figure 4. Average values of (a) $f_{\text {skpex }}$ and (b) $D_{\text {slope }}$ measured during injection of C. parvum into the QCM-D flow modules as a function of oocyst concentration: ( $\square$ ) in PBS alone, ( $\boldsymbol{\Delta}$ ) in the presence of $1 \mathrm{mg} / \mathrm{L}$ SRHA, and ( $\boldsymbol{m})$ in the presence of $1 \mathrm{mg} / \mathrm{L} \mathrm{SRFA}$. Error bars correspond to $95 \%$ confidence intervals for at least 3 separate experiments.
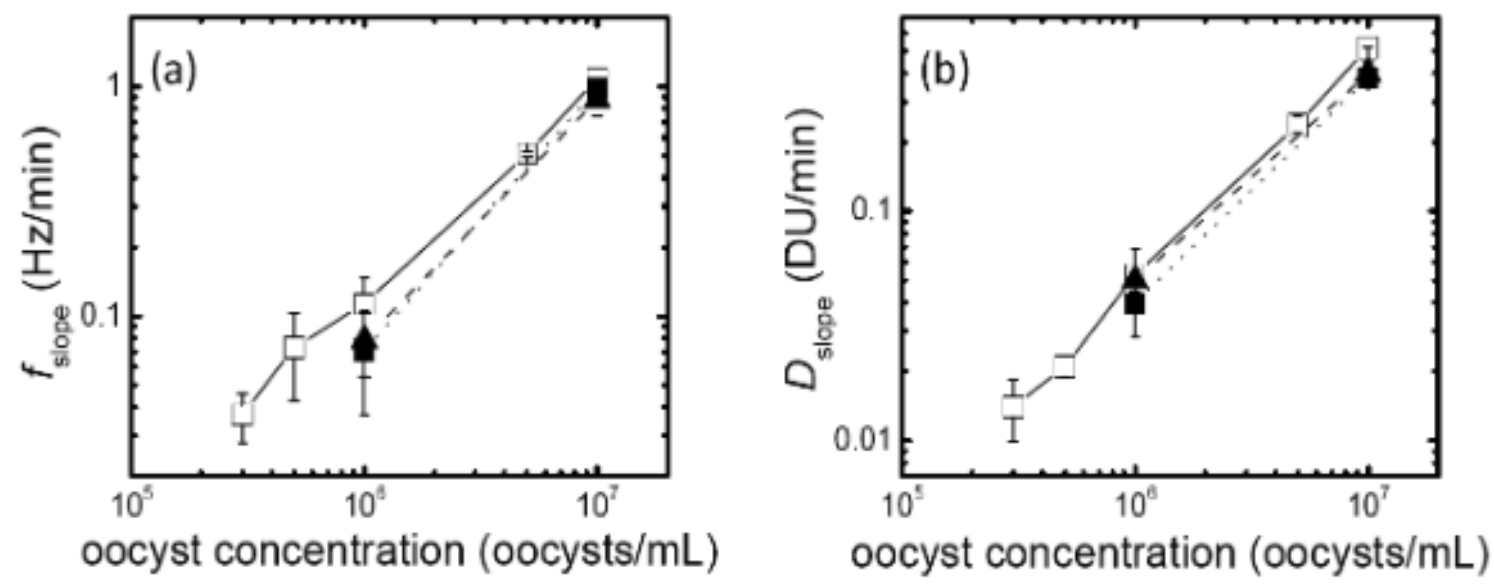

Various approaches have been used for detection of $C$. parvum oocysts in complex matrices. (Robinson et al., 2008) proposed a method allowing detection of less than 5 oocysts per gram of human feces. However, their approach necessitates sample preparation by immunomagnetic separation and oocyst labeling for detection by fluorescence microscopy. (Barbosa et al., 2008) used flow cytometry to detect $C$. parvum oocysts in stool with a sensitivity of $2 \times 10^{3}$ oocysts $/ \mathrm{mL}$, but their protocol also required sample preparation and labeling. Kang et al (Kang et al., 2008, Kang et al., 2006) employed surface plasmon resonance to achieve a sensitivity as low as $10^{2}$ oocysts/mL when various oocyst preparation methods were utilized. 
Recently, (Campbell and Mutharasan, 2008) reported rapid detection ( 1 min) and low detection limits for $C$. parvum (between $10^{2}$ to $10^{5}$ oocysts $/ \mathrm{mL}$ in a clean PBS matrix) by using piezoelectric cantilevers. QCM-D provides similar advantages to the piezoelectric cantilever approach. Use of the initial $f_{\text {slope }}$ and $D_{\text {slope }}$ as biosensor responses provide for rapid detection as these values are calculated from the frequency $(f)$ and energy dissipation $(D)$ measurements during the first few minutes of oocyst injection into the flow chamber. Using this approach, there is no need to wait for a stable plateau to be reached in the QCM-D response (i.e., the frequency or dissipation shifts). Hence, $C$. parvum detection can be confirmed very rapidly with this approach. Moreover, the proposed technique does not require labeling of the oocysts for detection.

A series of experiments were conducted to confirm that the measured $f$ and $D$ shifts correspond to specific binding of oocysts with the antibody layer on the crystal surface. These control experiments were conducted using crystals that were prepared without the addition of antibody. When these "no antibody" crystals were exposed to a suspension of C. parvum oocysts $\left(10^{7}\right)$, no measurable shifts in $f$ or $D$ were observed. In addition, following each experiment, the crystals were removed from the flow chambers, rinsed with DI water, dried, and viewed by optical microscopy. Figure 5a shows an optical microscopy image of a QCM-D crystal prepared with physisorbed antibody and BSA and exposed to C. parvum $\left(5 \times 10^{6}\right.$ oocyst $/ \mathrm{mL}$ ) for $60 \mathrm{~min}$. The image shows abundant coverage of $C$. parvum oocysts on the crystal surface. In contrast, the crystal prepared without antibody reveals very little binding of $C$. parvum on the sensing surface (Fig. $5 \mathrm{~b}$ ). Hence, the measured $f$ and $D$ shifts can be attributed to specific binding of the oocysts to the layer of physisorbed antibodies. 
Figure 5. 4x magnification light-microscopy images of (a) sensor prepared with antibody and BSA, and (b) sensor prepared without antibody after exposure to $60 \mathrm{~min}$ injection of C. parvum oocysts $\left(5 \times 10^{6}\right.$ oocysts $\left./ \mathrm{mL}\right)$. The negative of the microscope images are presented for improved clarity (i.e., oocysts are shown as black spots on a white background).
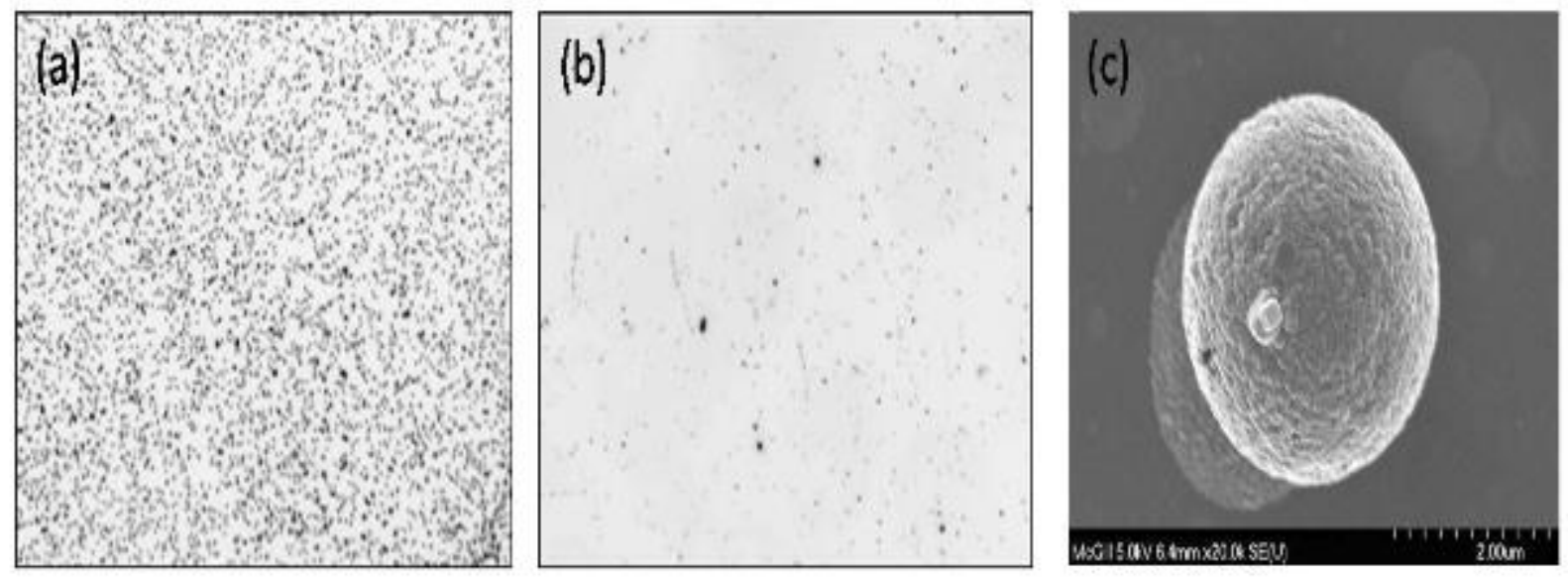


\subsection{Cryptosporidium Detection in the Presence of Nonbiological Interferents. Many}

studies reporting the development of biosensors for microbial pathogens such as C. parvum neglect the potential interference caused by other suspended particles (e.g., nonbiological colloids) or dissolved macromolecules such as organic acids or extracellular polymeric substances (Campbell and Mutharasan, 2008, Kang et al., 2006, Subramanian et al., 2006, Wong et al., 2002). We conducted QCM-D experiments to evaluate the potential interference caused by humic and fulvic acids which are common organic macromolecules found in surface waters and groundwaters that may be contaminated by microbial pathogens. The results of experiments conducted in the presence of $1 \mathrm{mg} / \mathrm{L}$ SRHA or SRFA are presented in Figure 4 as solid symbols. For the two tested concentrations of $C$. parvum, the biosensor response (i.e., measured values of $f_{\text {slope }}$ and $\left.D_{\text {slope }}\right)$ in the presence of the organic acids is generally very close to that obtained under "clean" conditions (open symbols); however, the response does decrease for some of the conditions (e.g., Fig. 4a, $1 \mathrm{mg} / \mathrm{L}$ SRFA). Humic and fulvic acids can adsorb onto the surface of microorganisms or nonbiological colloids (Dai and Hozalski, 2003, Franchi and O'Melia, 2003) resulting in increased negative charge. Indeed, many studies have shown that NOM can adsorb onto the surface of $C$. parvum oocysts thereby rendering them more negative (Dai and Hozalski, 2003, Tufenkji et al., 2006). This greater negative charge on the oocyst surface may lead to increased repulsive electrical double-layer interactions upon approach of the oocyst to the QCM crystal surface (Derjaguin and Landau, 1941, Verwey and Overbeek, 1948). Although this phenomenon may be partly responsible for the observed decrease in the biosensor signal at some conditions (Figure 4), it is more likely that steric stabilization plays an important role in the noted interference. Several other researchers have suggested that adsorption of NOM onto colloids (including C. parvum oocysts) may prevent colloid-surface interactions by a mechanism 
of steric stabilization (Franchi and O'Melia, 2003, Pelley and Tufenkji, 2008, Tufenkji et al., 2006). The organic acids may also interfere with $C$. parvum detection by masking antigen sites on the oocyst and/or binding sites on the immobilized layer of antibodies.

Latex microspheres are commonly used as model colloidal contaminants in studies related to water quality and treatment (Franchi and O'Melia, 2003, Li et al., 2004, Pelley and Tufenkji, 2008, Tufenkji et al., 2004). Suspensions of $C$. parvum $\left(5 \times 10^{6}\right.$ oocysts/mL $)$ were mixed with latex colloids $\left(1.5 \mu \mathrm{m} ; 5 \times 10^{6}\right.$ particles $\left./ \mathrm{mL}\right)$ and injected into the QCM-D flow chamber to evaluate the potential interference caused by the model colloidal contaminant. Values of $f_{\text {slope }}$ and $D_{\text {slope }}$ measured in the presence of the latex microspheres are presented in Figure 6 along with the biosensor responses for $C$. parvum alone $\left(10^{7}\right.$ oocysts $/ \mathrm{mL}$ and $5 \times 10^{6}$ oocysts/mL). The latex colloids cause significantly more interference than the organic acids, resulting in decreases of $64 \%$ and $61 \%$ in $f_{\text {slope }}$ and $D_{\text {slope, }}$ respectively, when compared to the experiment with $C$. parvum alone. 
Figure 6. Average values of (a) $f_{\text {slope }}$ and (b) $D_{\text {slope }}$ measured during injection of: (i) $10^{7}$ oocysts $/ \mathrm{mL}$ in PBS alone, (ii) $5 \times 10^{6}$ oocysts $/ \mathrm{mL}$ in PBS alone, (iii) $5 \times 10^{6}$ oocysts $/ \mathrm{mL}$ in a mixture with $E$. coli $0157: \mathrm{H} 7\left(5 \times 10^{6}\right.$ cells $\left./ \mathrm{mL}\right)$, (iv) $5 \times 10^{6}$ oocysts $/ \mathrm{mL}$ in a mixture with $E$. faecalis $\left(5 \times 10^{6}\right.$ cells $\left./ \mathrm{mL}\right)$, and (v) $5 \times 10^{6}$ oocysts $/ \mathrm{mL}$ in a mixture with $1.5 \mu \mathrm{m}$ latex microspheres $\left(5 \times 10^{6}\right.$ particles $\left./ \mathrm{mL}\right)$. Error bars correspond to $95 \%$ confidence intervals for at least 3 separate experiments.
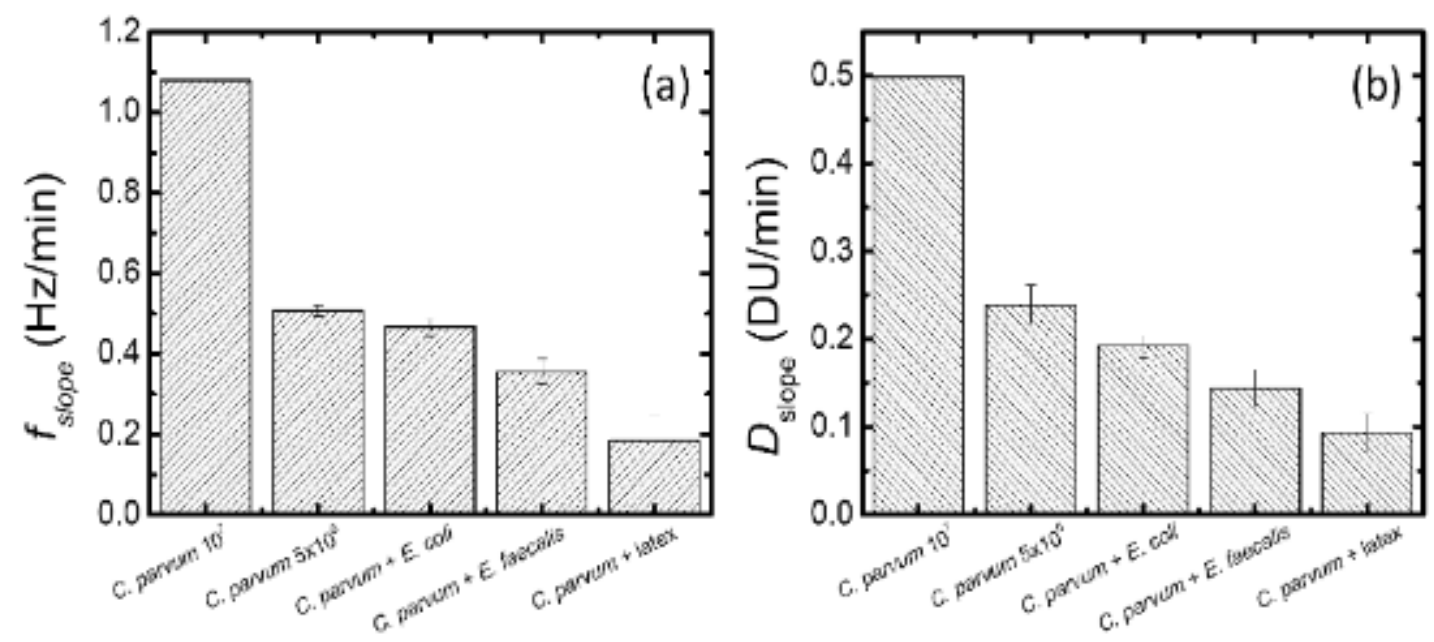


\subsection{Cryptosporidium Detection in the Presence of other Biological Agents. Water}

samples believed to be contaminated by an organism such as $C$. parvum may also contain a wide range of other organisms, including bacteria and viruses. Such organisms may cause interference during biosensing for the target parasite. The waterborne bacterial pathogens $E$. coli O157:H7 and E. faecalis were selected as representative Gram-negative and Gram-positive organisms, respectively, for evaluation of biosensor interference. When E. coli $\mathrm{O} 157: \mathrm{H} 7$ was injected alone onto the anti-C. parvum antibody functionalized biosensor surfaces, the measured $f$ and $D$ shifts were not distinguishable from the recognized instrument drift. It is also important to verify the biosensor response when $C$. parvum is mixed with bacteria. Values of $f_{\text {slope }}$ and $D_{\text {slope }}$ measured in the presence of either bacterium $\left(5 \times 10^{6}\right.$ cells $\left./ \mathrm{mL}\right)$ are presented in Figure 6 . The data shows that $E$. coli acts as a moderate interferent resulting in a decrease in the biosensor response; the value of $f_{\text {slope }}$ decreased by $8 \%$ whereas $D_{\text {slope }}$ decreased by $20 \%$ in the presence of E. coli $\mathrm{O} 157: \mathrm{H} 7$. The Gram-positive E. faecalis caused greater interference of the biosensor, resulting in a $30 \%$ decrease in $f_{\text {slope }}$ and a $40 \%$ decrease in $D_{\text {slope }}$ when compared to the experiment with C. parvum alone. The biosensor responses observed for the mixtures of bacteria and $C$. parvum suggest that the bacteria act as interferents even though they are not readily detected when injected alone into the biosensing flow modules. This result suggests that the bacteria may deposit onto the functionalized crystal surface, but not in large enough numbers to be detected. Although the deposited bacteria do not give rise to measurable changes in $f$ or $D$ when alone, they may affect detection of $C$. parvum by non-specifically binding to the crystal surface and thus effectively reducing (i.e., masking) the number of "free" antibody sites. This would explain the noted decrease in measured values of $f_{\text {slope }}$ and $D_{\text {slope }}$ in the presence of either bacterium (Figure 6). 


\section{Conclusions}

QCM-D was evaluated for detection of C. parvum via direct physisorption of antibodies onto gold-coated crystals. The initial $f_{\text {slope }}$ and $D_{\text {slope }}$ measured after injection of $C$. parvum yield similar linear working ranges $\left(3 \times 10^{5}\right.$ to $10^{7}$ oocysts $\left./ \mathrm{mL}\right)$ and rapid detection $(\sim 5$ min) without sample preparation or concentration. When oocysts were injected in the presence of humic or fulvic acids, the biosensor response was sometimes negatively affected; likely as a result of steric stabilization or masking of antibodies and/or antigens. More significant interference was observed when C. parvum was mixed with colloids, either biological (E. coli, E. faecalis) or nonbiological (latex microspheres). The model non-biological latex particles yielded the greatest extent of biosensor interference $(64 \%)$ whereas the Gram-negative bacterium (E. coli) has a significantly lower effect (i.e., the biosensor response decreased by only 20\%). The observed decrease in the biosensor response suggests that the mechanism of interference is one of nonspecific colloid deposition onto the antibody covered crystal surface. The detection limit of the proposed QCM-D based biosensor is not as low as that reported by other researchers working with PEMC sensing devices. Moreover, the detection limit of this and most other sensors is significantly higher than typical environmental concentrations of $C$. parvum observed in surface waters ( 1 oocyst/mL) (Ajeagah et al., 2007, Mons et al., 2009). Further studies are thus needed to significantly improve the biosensor response for parasite detection in complex water matrices containing lower oocyst numbers. Some techniques that may yield improved biosensor responses include (i) use of crystals with a higher fundamental resonance frequency, (ii) higherdensity packing of the antibodies on the crystal surface and (iii) nanoparticle amplification. The 
results of this study underscore the importance of evaluating the potential interference of biosensors using both biological and non-biological agents. 


\section{Acknowledgements}

This research was supported by NSERC (Discovery Grant and PGS awarded to C.P.), the Canada Research Chairs Program, the FQRNT Centre for Biorecognition and Biosensors (CBB), the Canadian Water Network (CWN), and the Canada Foundation for Innovation (CFI). 


\section{References}

Ajeagah, G., Njine, T., Foto, S., Bilong Bilong, C.F. and Karanis, P., 2007. Enumeration of Cryptosporidium spp and Giardia spp (oo)cysts in a tropical eutrophic lake: The municipal lake of Yaounde. Int. J. Environ. Sci. Techn. 4(2), 223-232.

Barbosa, J.M.M., Costa-de-Oliveira, S., Rodrigues, A.G., Hanscheid, T., Shapiro, H. and Pina-Vaz, C., 2008. A flow cytometric protocol for detection of Cryptosporidium spp. Cytometry, Part A 73A(1), 4447.

Campbell, G.A. and Mutharasan, R., 2008. Near real-time detection of Cryptosporidium parvum oocyst by IgM-functionalized piezoelectric-excited millimeter-sized cantilever biosensor. Biosensors \& Bioelectronics 23(7), 1039-1045.

Campbell, G.A., Uknalis, J., Tu, S.-I. and Mutharasan, R., 2007. Detect of Escherichia coli O157:H7 in ground beef samples using piezoelectric excited millimeter-sized cantilever (PEMC) sensors. Biosensors \& Bioelectronics 22(7), 1296-1302.

Casemore, D.P., Wright, S.E. and Coop, R.L. (1997) Cryptosporidium and Cryptosporidiosis. Fayer, R. (ed), pp. 65-92, CRC Press, Boca Raton, FL.

Dai, X. and Hozalski, R.M., 2003. Evaluation of Microspheres as Surrogates for Cryptosporidium parvum Oocysts in Filtration Experiments. Environmental Science and Technology 37(5), 1037-1042.

Derjaguin, B.V. and Landau, L.D., 1941. Theory of the stability of strongly charged lyophobic sols and of the adhesion of strongly charged particles in solutions of electrolytes. Acta Physicochim. URSS 14, 733762.

Dolatshahi-Pirouz, A., Rechendorff, K., Hovgaard, M.B., Foss, M., Chevallier, J. and Besenbacher, F., 2008. Bovine serum albumin adsorption on nano-rough platinum surfaces studied by QCM-D. Colloids and Surfaces, B: Biointerfaces 66(1), 53-59.

Edgar, R., McKinstry, M., Hwang, J., Oppenheim, A.B., Fekete, R.A., Giulian, G., Merril, C., Nagashima, K. and Adhya, S., 2006. High-sensitivity bacterial detection using biotin-tagged phage and quantum-dot nanocomplexes. Proceedings of the National Academy of Sciences of the United States of America 103(13), 4841-4845.

Fatisson, J., Domingos, R.F., Wilkinson, K.J. and Tufenkji, N., 2009. Deposition Kinetics of TiO2 Nanoparticles onto Silica Measured using QCM-D. Langmuir (under review).

Franchi, A. and O'Melia, C.R., 2003. Effects of Natural Organic Matter and Solution Chemistry on the Deposition and Reentrainment of Colloids in Porous Media. Environmental Science and Technology 37(6), 1122-1129.

Green, R.J., Davies, J., Davies, M.C., Roberts, C.J. and Tendler, S.J.B., 1997. Surface plasmon resonance for real time in situ analysis of protein adsorption to polymer surfaces. Biomaterials 18(5), 405-413.

Hook, F. (1997) Development of a novel QCM technique for protein adsorption studies, in Department of Biochemistry and Biophysics and Department of Applied Physics, Chalmers University of Technology.

Hook, F., Kasemo, B., Nylander, T., Fant, C., Sott, K. and Elwing, H., 2001. Variations in coupled water, viscoelastic properties, and film thickness of a Mefp-1 protein film during adsorption and cross-linking: A quartz crystal microbalance with dissipation monitoring, ellipsometry, and surface plasmon resonance study. Analytical Chemistry 73(24), 5796-5804.

Hook, F., Rodahl, M., Brzezinski, P. and Kasemo, B., 1998. Energy Dissipation Kinetics for Protein and Antibody-Antigen Adsorption under Shear Oscillation on a Quartz Crystal Microbalance. Langmuir 14(4), 729-734.

Hook, F., Voros, J., Rodahl, M., Kurrat, R., Boni, P., Ramsden, J.J., Textor, M., Spencer, N.D., Tengvall, P., Gold, J. and Kasemo, B., 2002. A comparative study of protein adsorption on titanium oxide surfaces using in situ ellipsometry, optical waveguide lightmode spectroscopy, and quartz crystal microbalance/dissipation. Colloids and Surfaces, B: Biointerfaces 24(2), 155-170. 
Jex, A.R., Smith, H.V., Monis, P.T., Campbell, B.E. and Gasser, R.B., 2008. Cryptosporidium Biotechnological advances in the detection, diagnosis and analysis of genetic variation. Biotechnology Advances 26, 304-317.

Kang, C.D., Cao, C., Lee, J., Choi, I.S., Kim, B.W. and Sim, S.J., 2008. Surface plasmon resonance-based inhibition assay for real-time detection of Cryptosporidium parvum oocyst. Water Research 42(6-7), 1693-1699.

Kang, C.D., Lee, S.W., Park, T.H. and Sim, S.J., 2006. Performance enhancement of real-time detection of protozoan parasite, Cryptosporidium oocyst by a modified surface plasmon resonance (SPR) biosensor. Enzyme and Microbial Technology 39(3), 387-390.

Kim, J. and Somorjai, G.A., 2003. Molecular Packing of Lysozyme, Fibrinogen, and Bovine Serum Albumin on Hydrophilic and Hydrophobic Surfaces Studied by Infrared-Visible Sum Frequency Generation and Fluorescence Microscopy. Journal of the American Chemical Society 125(10), 31503158.

Lee, S.-H., Stubbd, D.D., Cairney, J. and Hunt, W.D., 2005. Rapid Detection of Bacterial Spores Using a Quartz Crystal Microbalance (QCM) Immunoassay. IEEE Sensors Journal 5(4), 737-743.

Li, X., Scheibe, T.D. and Johnson, W.P., 2004. Apparent Decreases in Colloid Deposition Rate Coefficients with Distance of Transport under Unfavorable Deposition Conditions: A General Phenomenon. Environmental Science \& Technology 38, 5616-5625.

Lisle, J.T. and Rose, J.B., 1995. Cryptosporidium Contamination of Water in the USA and UK - a Minireview. Journal of Water Supply Research and Technology-Aqua 44(3), 103-117.

Lubarsky, G.V., Davidson, M.R. and Bradley, R.H., 2007. Hydration-dehydration of adsorbed protein films studied by AFM and QCM-D. Biosensors \& Bioelectronics 22(7), 1275-1281.

Mao, X., Yang, L., Su, X.L. and Li, Y., 2006. A nanoparticle amplification based quartz crystal microbalance DNA sensor for detection of Escherichia coli O157:H7. Biosensors and Bioelectronics 21, $1178-1185$.

Mons, C., Dumetre, A., Gosselin, S., Galliot, C. and Moulin, L., 2009. Monitoring of Cryptosporidium and Giardia river contamination in Paris area. Water Research 43, 211-217.

Nimeri, G., Fredriksson, C., Elwing, H., Liu, L., Rodahl, M. and Kasemo, B., 1998. Neutrophil interaction with protein-coated surfaces studied by an extended quartz crystal microbalance technique. Colloids and Surfaces, B: Biointerfaces 11(5), 255-264.

Pelley, A.J. and Tufenkji, N., 2008. Effect of particle size and natural organic matter on the migration of nano- and microscale latex particles in saturated porous media. Journal of Colloid and Interface Science 321, 74-83.

Poitras, C. and Tufenkji, N., 2009. A QCM-D Based Biosensor for E. coli O157:H7 Highlighting the Relevance of the Dissipation Slope as a Transduction Signal. Biosens Bioelectron 24, 2137-2142.

Robinson, G., Watkins, J. and Chalmers, R.M., 2008. Evaluation of a modified semi-automated immunomagnetic separation technique for the detection of Cryptosporidium oocysts in human faeces. Journal of microbiological methods 75(1), 139-141.

Rose, J.B., 1997. Environmental ecology of Cryptosporidium and public health implications. Annual Review of Public Health 18, 135-161.

Sauerbrey, G.Z., 1959. The use of oscillators for weighing thin layers and for microweighing. Z. Phys. $155,2333-2336$.

Shen, Z., Huang, M., Xiao, C., Zhang, Y., Zeng, X. and Wang, P.G., 2007. Nonlabeled Quartz Crystal Microbalance Biosensor for Bacterial Detection Using Carbohydrate and Lectin Recognitions. Anal. Chem. 79, 2312-2319.

Smith, H.V., 1992. Cryptosporidium and Water - a Review. Journal of the Institution of Water and Environmental Management 6(4), 443-451.

Smith, J.E. and Perdek, J.M., 2004. Assessment and management of watershed microbial contaminants. Critical Reviews in Environmental Science and Technology 34, 109-139. 
Su, X.-L. and Li, Y., 2005. A QCM immunosensor for Salmonella detection with simultaneous measurements of resonant frequency and motional resistance. Biosensors \& Bioelectronics 21(6), 840848.

$\mathrm{Su}$, X.L. and Li, Y., 2004. A self-assembled monolayer-based piezoelectric immunosensor for rapid detection of Escherichia coli O157:H7. Biosensors and Bioelectronics 19(6), 563-574.

Subramanian, A., Irudayaraj, J. and Ryan, T., 2006. A mixed self-assembled monolayer-based surface plasmon immunosensor for detection of E. coli O157:H7. Biosensors \& Bioelectronics 21(7), 998-1006.

Tencer, M., Charbonneau, R., Lahoud, N. and Berini, P., 2007. AFM study of BSA adlayers on Au stripes. Applied Surface Science 253, 9209-9214.

Tufenkji, N., Dixon, D.R., Considine, R. and Drummond, C.J., 2006. Multi-scale Cryptosporidium/sand interactions in water treatment. Water Research 40(18), 3315-3331.

Tufenkji, N., Miller, G.F., Ryan, J.N., Harvey, R.W. and Elimelech, M., 2004. Transport of Cryptosporidium Oocysts in Porous Media: Role of Straining and Physicochemical Filtration. Environmental Science \& Technology 38, 5932-5938.

Vaughan, R.D., O'Sullivan, C.K. and Guilbault, G.G., 2001. Development of a quartz crystal microbalance (QCM) immunosensor for the detection of Listeria monocytogenes. Enzyme and Microbial Technology 29(10), 635-638.

Verwey, E.J.W. and Overbeek, J.T.G., 1948. Theory of the Stability of Lyophobic Colloids, Elsevier, Amsterdam.

Waswa, J., Irudayaraj, J. and DebRoy, C., 2006. Direct detection of E. coli O157:H7 in selected food systems by a surface plasmon resonance biosensor. LWT--Food Science and Technology 40(2), 187-192.

Wong, Y.Y., Ng, S.P., Ng, M.H., Si, S.H., Yao, S.Z. and Fung, Y.S., 2002. Immunosensor for the differentiation and detection of Salmonella species based on a quartz crystal microbalance. Biosensors and Bioelectronics 17(8), 676-684. 